\title{
Noncoherent Sequence Detection in Frequency Nonselective Slowly Fading Channels
}

\author{
Giulio Colavolpe, Student Member, IEEE, and Riccardo Raheli, Member, IEEE
}

\begin{abstract}
A new class of noncoherent sequence detection (NSD) algorithms for combined demodulation and decoding of any coded linear and continuous phase modulations, transmitted over additive white Gaussian noise (AWGN) channels, has been recently presented. In this paper, this class is generalized to the case of frequency nonselective Rayleigh or Rice slowly fading channels, in the presence or absence of channel state information. Coded linear modulations, namely $M$-ary phase shift keying $(M$-PSK) and quadrature amplitude modulation ( $M$-QAM), are considered. The proposed detection schemes have a performance which approaches that of coherent detectors, are very robust to phase and frequency instabilities, and compare favorably to other solutions previously proposed in the technical literature.
\end{abstract}

Index Terms-Fading channels, maximum likelihood decoding, noncoherent sequence detection, Viterbi decoding.

\section{INTRODUCTION}

$\mathbf{I}$ N THE technical literature, a growing effort has been recently devoted toward the derivation of improved noncoherent detection or decoding schemes [1]-[16]. Improvements with respect to differential receivers [17] were proposed in [3]-[6] for PSK modulations, using an extended differential detector based on decision feedback. Multiple-symbol differential detection (MSDD) was presented in [7]-[9] for PSK modulation (see also [10]) and extended to trellis-coded PSK [11]-[13], coded and uncoded quadrature amplitude modulation (QAM), and fading channels [14], and to improve the performance of coherent receivers based on a phase-locked loop (PLL) [15]. Multiple-symbol differential receivers are based on maximum likelihood detection of a block of information symbols based on the observation of a corresponding signal block. A trellis-based noncoherent detection scheme is considered in [16], which makes use of a Viterbi algorithm whose branch metrics are heuristically assumed equal to the block metrics in MSDD. As a general result, the performance of noncoherent schemes based on extended windows of observation of the received signal improves, for increasing observation length and receiver complexity, and rapidly approaches that of optimal coherent receivers.

In [1], NSD schemes for combined detection and decoding of coded linear modulations have been proposed. This approach "moves beyond the crude block detection found in this area, to more natural, logical and optimal trellis-based detection

\footnotetext{
Manuscript received March 27, 1999; revised April 18, 2000. This work was supported by Ministero dell'Università e della Ricerca Scientifica e Tecnologica (MURST), Italy. This paper was presented in part at the Global Telecommunications Conference (GLOBECOM'99), Rio de Janeiro, Brazil, December 1999.

The authors are with the Dipartimento di Ingegneria dell'Informazione, Università di Parma, Viale delle Scienze, 43100 Parma, Italy.

Publisher Item Identifier S 0733-8716(00)09209-X.
}

schemes." ${ }^{1}$ The starting point in [1] is the optimal noncoherent receiver which operates in the presence of a random phase rotation of the received signal, modeled as constant during the transmission, and AWGN. Since optimal sequence detection requires a search of a path in a tree diagram, the required complexity increases exponentially with the duration of the transmission. In [1], proper approximations are proposed in order to reduce the problem to a search of a path in a trellis diagram and realize simple suboptimal detection schemes based on a Viterbi algorithm. Besides being realizable, these suboptimal schemes have the convenient feature of allowing us to remove the constant phase assumption and encompassing time-varying phase models.

For increasing complexity, the performance of NSD schemes is shown to approach that of optimal coherent receivers [1], [18]. A performance gain may be obtained with respect to existing schemes, with acceptable levels of complexity-the tradeoff between complexity and performance being simply controlled by a parameter referred to as implicit phase memory and the number of trellis states [1]. NSD schemes are very robust to oscillator instabilities, such as phase noise and time-varying frequency offsets, and do not require an acquisition period as in the case of coherent detection schemes based on PLLs. For these reasons, they appear very attractive for burst-mode transmissions typical of future generation wireless local loop, mobile-to-satellite, and cellular mobile radio systems.

In this paper, noncoherent sequence detection is extended to the case of Rayleigh or Rice frequency nonselective slowly fading channels. To this purpose, two approaches are considered. In the first approach, the problem of noncoherent sequence detection under the assumption of perfect knowledge of the amplitude of the channel fading is faced. We refer to this case as detection in the presence of channel state information (CSI). In the second approach, the detection problem in the absence of knowledge of the fading amplitude is addressed. Although in principle a statistical knowledge of the channel fading amplitude would be necessary, excellent approximations are obtained which do not require any knowledge of the channel statistics. We refer to this case as detection in the absence of CSI. Although the paper concentrates on coded linear modulations, extensions to the case of continuous phase modulations may be dealt with by the methods described in [1] and [2].

The proposed algorithms are very effective for Rayleigh or Rice fading and AWGN channels. Numerical results for multiamplitude modulation formats, such as 16-QAM, are provided, showing that the proposed NSD receivers exhibit minor energy losses with respect to ideal coherent detectors. These schemes compare very favorably to previously proposed

${ }^{1}$ Quoted from an anonymous review of [1]. 


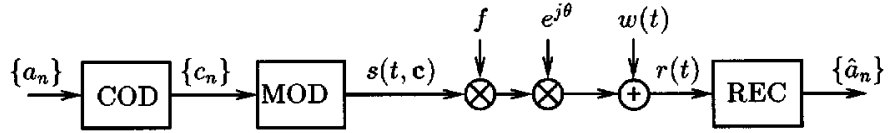

Fig. 1. System model.

ones and, to our knowledge, are the only detection schemes of practical significance for multiamplitude modulations. In [14], the problem of MSDD of QAM signals transmitted over slow Rayleigh fading channels is considered. Although specific solutions are proposed in [14], their performance is not analyzed. We show that the metric proposed in [14] for this detection problem yields a significant irreducible error probability, or error floor. This result, observed by means of simulation, is theoretically proved in the Appendix.

In the next section, we review the assumed system model and relevant background. The proposed NSD schemes in the presence of CSI are described in Section III. In Section IV, the case of absence of CSI is considered. The resulting branch metrics depend on some statistical parameters of the fading amplitude. Approximations are then proposed in order to obtain simplified branch metrics which do not require knowledge (or estimation) of any statistical channel parameter. In Section V, numerical results are presented. For increasing complexity, the performance of the proposed detection schemes is shown to approach that of optimal coherent receivers. Finally, conclusions are drawn in Section VI.

\section{SySTEM MODEL AND BACKGROUND}

The assumed system model is shown in Fig. 1. An information sequence $\left\{a_{n}\right\}$, composed of independent and identically distributed symbols belonging to an $M$-ary alphabet, is mapped into a code sequence by means of some coding rule. We assume a burst-mode transmission with $K$ code symbols per burst and denote with $\boldsymbol{c}=\left(c_{0}, c_{1}, \cdots, c_{K-1}\right)^{T}$ a column vector whose elements are the code symbols transmitted in a burst. The code sequence is further mapped by a modulator into a time-continuous signal $s(t, c)$ which is transmitted over a frequency nonselective slowly fading channel, represented by a multiplicative complex random variable $f$, modeled as Gaussian with mean $\eta_{f}$ and variance $\sigma_{f}^{2}$. The transmitted signal also undergoes a phase rotation $\theta$, modeled as a random variable with uniform distribution in the interval $[0,2 \pi)$ and independent of $f$. Although both $f$ and $\theta$ are assumed to be constant during the transmission of each data burst, the approximation of memory truncation introduced in the next sections, as in [1] and [2], has the convenient feature of allowing us to remove the constant channel assumption and encompass time-varying models.

The choice of the value of $\eta_{f}$ allows us to take into account both Rayleigh $\left(\eta_{f}=0\right)$ and Rice fading distributions, with a factor $K_{R} \triangleq\left|\eta_{f}\right|^{2} / \sigma_{f}^{2}$. The channel also introduces AWGN of complex envelope $w(t)$ with independent real and imaginary components, each with two-sided power spectral density $N_{0}$. Assuming $\eta_{f}=1$ and $\sigma_{f}^{2}=0$, we obtain the limiting case of an AWGN channel.

Assuming that signal $s(t, c)$ is linearly modulated, it may be easily shown that sampling the output of a filter matched to the shaping pulse, with one sample per signaling interval, yields a sufficient statistic for optimal detection of the information sequence. ${ }^{2}$ In the absence of intersymbol interference, this sampled output may be expressed as

$$
x_{n}=f e^{j \theta} c_{n}+w_{n}
$$

where $\left\{w_{n}\right\}$ are independent identically distributed complex noise samples with independent components, each with variance $\sigma^{2}$. In the following, the samples $\left\{x_{n}\right\}$ will be referred to as observations.

\section{NSD WITH PERFECT CHANNEL StATE INFORMATION}

We now assume that the receiver has perfect CSI, represented by the channel gain $|f|$. As an example, this knowledge may be well approximated by means of a simple data-aided estimate based on a known preamble preceding the data burst. In the case of perfect CSI, an alternative sufficient statistic may be obtained by normalizing the received samples $\left\{x_{n}\right\}$ using the known gain value as

$$
x_{n}^{\prime} \triangleq \frac{x_{n}}{|f|}
$$

Using polar coordinates to express the fading coefficient as $f=$ $|f| e^{j \alpha}$, we have

$$
x_{n}^{\prime}=c_{n} e^{j \theta^{\prime}}+w_{n}^{\prime}
$$

where

$$
\begin{aligned}
\theta^{\prime} & \triangleq \theta+\alpha \\
w_{n}^{\prime} & \triangleq \\
|f| & w_{n}
\end{aligned}
$$

With respect to a channel which introduces an unknown phase shift only, considered in detail in [1] and [2], we now have two differences: the phase shift is $\theta^{\prime}$ and the variance of the noise samples is $\sigma^{2} /|f|^{2}$. The random variable $\theta^{\prime}$ has uniform distribution, independently of the distribution of $\alpha$. Under the approximations used in [1] and [2], the noise variance does not affect the receiver strategy. Therefore, the results in [1] and [2], still apply and the NSD receiver, in the case of perfect CSI, may be based on a Viterbi algorithm with branch metrics

$$
\lambda_{n}(\tilde{\boldsymbol{c}})=\left|\sum_{k=0}^{N-1} x_{n-k}^{\prime} \tilde{c}_{n-k}^{*}\right|-\left|\sum_{k=1}^{N-1} x_{n-k}^{\prime} \tilde{c}_{n-k}^{*}\right|-\frac{1}{2}\left|\tilde{c}_{n}\right|^{2}
$$

where $\tilde{\boldsymbol{c}}$ is a hypothetical code vector and $N$ is an integer which is referred to as (implicit) phase memory, as in [1] and [2].

\section{NSD Without ChanNEL STATE INFORMATION}

In this section, we derive the NSD receiver assuming absence of CSI, in the sense that the receiver has a statistical information about the fading gain only. Specifically, using polar coordinates to express the mean $\eta_{f}$ of the random variable $f$ as $\eta_{f}=\left|\eta_{f}\right| e^{j \theta_{f}}$, we assume that the receiver knows the value

\footnotetext{
${ }^{2}$ This is not true in the case of a time-varying fading channel, where a sufficient statistic may require the use of oversampling [19].
} 
$\left|\eta_{f}\right|$, the fading variance $\sigma_{f}^{2}$, and the noise variance $\sigma^{2}$. In principle, an estimation of these parameters could be obtained from the observation of a few preambles of consecutives bursts. However, it is shown in the following that in all significant cases an estimation of these parameters is not necessary.

Recalling (1), the conditional probability density function of the observation vector $\boldsymbol{x} \triangleq\left(x_{0}, x_{1}, \cdots, x_{K-1}\right)^{T}$, given a code vector $\tilde{c}$ and a hypothetical phase $\tilde{\theta}$, is obviously Gaussian

$$
p(\boldsymbol{x} \mid \tilde{\boldsymbol{c}}, \tilde{\theta})=\frac{1}{\pi^{K} \operatorname{det}\left(\boldsymbol{C}_{\boldsymbol{x}}\right)} \exp \left[-\left(\boldsymbol{x}-\boldsymbol{\eta}_{x}\right)^{H} \boldsymbol{C}_{\boldsymbol{x}}^{-1}\left(\boldsymbol{x}-\boldsymbol{\eta}_{\boldsymbol{x}}\right)\right]
$$

where $[\cdot]^{H}$ is the transpose conjugate operator and

$$
\begin{aligned}
\boldsymbol{\eta}_{\boldsymbol{x}} & \triangleq E\{\boldsymbol{x} \mid \tilde{\boldsymbol{c}}, \tilde{\theta}\}=\eta_{f} e^{j \tilde{\theta} \tilde{\boldsymbol{c}}} \\
\boldsymbol{C}_{\boldsymbol{x}} & \triangleq E\left\{\left(\boldsymbol{x}-\boldsymbol{\eta}_{\boldsymbol{x}}\right)\left(\boldsymbol{x}-\boldsymbol{\eta}_{\boldsymbol{x}}\right)^{H} \mid \tilde{\boldsymbol{c}}, \tilde{\theta}\right\} \\
& =\sigma_{f}^{2} \tilde{\tilde{\boldsymbol{c}}} \tilde{c}^{H}+2 \sigma^{2} \boldsymbol{I}
\end{aligned}
$$

are the conditional mean and covariance matrix. Using [20, equation (3.54)], it is possible to express the inverse covariance matrix as

$$
\boldsymbol{C}_{\boldsymbol{x}}^{-1}=\left(\sigma_{f}^{2} \tilde{\boldsymbol{c}} \tilde{\boldsymbol{c}}^{H}+\sigma^{2} \boldsymbol{I}\right)^{-1}=\frac{1}{2 \sigma^{2}} \boldsymbol{I}-\frac{\sigma_{f}^{2}}{4 \sigma^{4}} \frac{\tilde{\boldsymbol{c}} \tilde{\boldsymbol{c}}^{H}}{1+\frac{\sigma_{f}^{2}}{2 \sigma^{2}}|\tilde{\boldsymbol{c}}|^{2}} .
$$

The following relation may also be easily verified:

$$
\operatorname{det}\left(\sigma_{f}^{2} \widetilde{\boldsymbol{c}} \tilde{\boldsymbol{c}}^{H}+2 \sigma^{2} \boldsymbol{I}\right)=\left(2 \sigma^{2}\right)^{K-1}\left(\sigma_{f}^{2}|\tilde{\boldsymbol{c}}|^{2}+2 \sigma^{2}\right) .
$$

Substituting (10) and (11) into (7), after some tedious but straightforward manipulations, we obtain (12) shown at the bottom of the page, which, in the last step, has been expressed as a function of $\gamma \triangleq \sigma_{f}^{2} / 2 \sigma^{2}$. This quantity is useful because the branch metrics derived below from (12) can be expressed as a function of $1 / \gamma$ in order to avoid numerical problems for high signal-to-noise ratio, when $\gamma \rightarrow+\infty$. Averaging (12) with respect to $\tilde{\theta}$, we obtain

$$
\begin{aligned}
p(\boldsymbol{x} \mid \tilde{\boldsymbol{c}})= & \frac{1}{2 \pi} \int_{0}^{2 \pi} p(\boldsymbol{x} \mid \tilde{\boldsymbol{c}}, \tilde{\theta}) d \tilde{\theta} \\
= & \frac{1}{\pi^{K}\left(2 \sigma^{2}\right)^{K-1} \sigma_{f}^{2}} \exp \left(-\frac{|\boldsymbol{x}|^{2}}{2 \sigma^{2}}\right) \\
& \cdot \frac{1}{|\tilde{\boldsymbol{c}}|^{2}+\frac{1}{\gamma}} \exp \left\{\frac{1}{2 \sigma^{2}}\left[\frac{\left|\tilde{\boldsymbol{c}}^{H} \boldsymbol{x}\right|^{2}-\frac{\left|\eta_{f}\right|^{2}|\tilde{\boldsymbol{c}}|^{2}}{\gamma}}{\frac{1}{\gamma}+|\tilde{\boldsymbol{c}}|^{2}}\right]\right\} \\
& \cdot I_{0}\left(\frac{1}{\sigma^{2}}\left[\frac{\left|\eta_{f}\right|\left|\tilde{\boldsymbol{c}}^{H} \boldsymbol{x}\right|}{\gamma}\right]\right)
\end{aligned}
$$

where $I_{0}(x)$ is the zeroth-order modified Bessel function of the first kind. For Rayleigh fading, (7) is independent of $\tilde{\theta}$. As a consequence, this averaging operation is not necessary and the $I_{0}(\cdot)$ function does not arise. Setting $\eta_{f}=0$ in (13) directly accounts for this fact. As intuitively expected, due to the noncoherent nature of the strategy, the result in (13) is independent of $\theta_{f}$. Therefore, knowledge of this phase is irrelevant for the detection process. Ideal NSD corresponds to the maximization of the following metric:

$$
\begin{aligned}
\Lambda_{K}(\tilde{\boldsymbol{c}}) \triangleq & \ln p(\boldsymbol{x} \mid \tilde{\boldsymbol{c}}) \\
\sim & -\sigma^{2} \ln \left(|\tilde{\boldsymbol{c}}|^{2}+\frac{1}{\gamma}\right)+\frac{1}{2}\left[\frac{\left|\tilde{\boldsymbol{c}}^{H} \boldsymbol{x}\right|^{2}-\frac{\left|\eta_{f}\right|^{2}|\tilde{\boldsymbol{c}}|^{2}}{\gamma}}{\frac{1}{\gamma}+|\tilde{\boldsymbol{c}}|^{2}}\right] \\
& +\sigma^{2} \ln I_{0}\left(\frac{1}{\sigma^{2}}\left[\frac{\frac{\left|\eta_{f}\right|\left|\tilde{\boldsymbol{c}}^{H} \boldsymbol{x}\right|}{\gamma}}{\frac{1}{\gamma}+|\tilde{\boldsymbol{c}}|^{2}}\right]\right)
\end{aligned}
$$

$$
\begin{aligned}
p(\boldsymbol{x} \mid \tilde{\boldsymbol{c}}, \tilde{\theta})= & \frac{1}{\pi^{K}\left(2 \sigma^{2}\right)^{K-1} \sigma_{f}^{2}} \frac{1}{|\tilde{\boldsymbol{c}}|^{2}+\frac{2 \sigma^{2}}{\sigma_{f}^{2}}} \exp \left(-\frac{|\boldsymbol{x}|^{2}}{2 \sigma^{2}}\right) \\
& \cdot \exp \left\{-\frac{1}{2 \sigma^{2}}\left[\frac{2 \frac{\sigma^{2}}{\sigma_{f}^{2}}\left|\eta_{f}\right|^{2}|\tilde{\boldsymbol{c}}|^{2}-\left|\tilde{\boldsymbol{c}}^{H} \boldsymbol{x}\right|^{2}-4 \frac{\sigma^{2}}{\sigma_{f}^{2}} \operatorname{Re}\left\{\eta_{f}^{*} e^{\left.-j \tilde{\theta} \tilde{\boldsymbol{c}}{ }^{H} \boldsymbol{x}\right\}}\right.}{\frac{2 \sigma^{2}}{\sigma_{f}^{2}}+|\tilde{\boldsymbol{c}}|^{2}}\right]\right\} \\
= & \frac{1}{\pi^{K}\left(2 \sigma^{2}\right)^{K-1} \sigma_{f}^{2}} \exp \left(-\frac{|\boldsymbol{x}|^{2}}{2 \sigma^{2}}\right) \frac{1}{|\tilde{\boldsymbol{c}}|^{2}+\frac{1}{\gamma}} \\
& \cdot \exp \left\{-\frac{1}{2 \sigma^{2}}\left[\frac{\frac{1}{\gamma}\left|\eta_{f}\right|^{2}|\tilde{\boldsymbol{c}}|^{2}-\left|\tilde{\boldsymbol{c}}^{H} \boldsymbol{x}\right|^{2}-\frac{2\left|\eta_{f}\right|\left|\tilde{\boldsymbol{c}}^{H} \boldsymbol{x}\right|}{\gamma} \cos \left(\arg \left\{\tilde{\boldsymbol{c}}^{H} \boldsymbol{x}\right\}-\theta_{f}-\tilde{\theta}\right)}{\frac{1}{\gamma}+|\tilde{\boldsymbol{c}}|^{2}}\right]\right\}
\end{aligned}
$$


where the expression $x \sim y$ denotes that $x$ and $y$ are monotonically related quantities. This metric is a special case of $[14$, equation (A-12)].

Equation (14) represents the exact expression of the likelihood function. Some approximations are now introduced in order to realize simple suboptimal detection schemes based on a Viterbi algorithm. We adopt the approximation $\ln \left[I_{0}(x)\right] \simeq x$, valid for large values of the argument. This approximation is valid for high values of $\left|\eta_{f}\right|$ and $1 / \sigma^{2}$, hence for high signal-tonoise ratio and a high Rice factor $K_{R}$. With this approximation, (14) may be expressed as shown in (15) at the bottom of the page. Proceeding as in [1] and [2], we may define a partial sequence metric at the $n$th signaling interval

$$
\begin{aligned}
& \Lambda_{n}(\tilde{\boldsymbol{c}}) \\
& \triangleq-\sigma^{2} \ln \left(\sum_{k=0}^{n-1}\left|\tilde{c}_{k}\right|^{2}+\frac{1}{\gamma}\right) \\
& \quad+\frac{1}{2} \frac{\left|\sum_{k=0}^{n-1} x_{k} \tilde{c}_{k}^{*}\right|^{2}-\frac{\left|\eta_{f}\right|^{2}}{\gamma} \sum_{k=0}^{n-1}\left|\tilde{c}_{k}\right|^{2}+2 \frac{\left|\eta_{f}\right|}{\gamma}\left|\sum_{k=0}^{n-1} x_{k} \tilde{c}_{k}^{*}\right|}{\frac{1}{\gamma}+\sum_{k=0}^{n-1}\left|\tilde{c}_{k}\right|^{2}}
\end{aligned}
$$

and an incremental metric as shown in (17) at the bottom of the page.
The difficulty inherent in the incremental metric (17) is its unlimited memory. In fact, this metric depends on the entire previous code sequence. This implies that the maximization of the sequence metric may, in principle, be realized by a search of a path in a properly defined tree diagram. From the implementation viewpoint, approximate tree search algorithms must be used, unless a very short transmission length is assumed. As in [1], in order to limit the memory of the incremental metric (17), a truncation is introduced, aimed at allowing us to search a trellis diagram by means of a Viterbi algorithm. To this end, in (17) we may consider $N \ll K$ most recent observations $x_{k}$ and code symbols $\tilde{c}_{k}$ only. After an initial transient period, i.e., for $n \geq N-1$, the resulting approximate truncated-memory incremental, or branch, metric is shown in (18) at the bottom of the next page. As already mentioned, the receiver is based on a Viterbi algorithm and requires knowledge of the parameters $\left|\eta_{f}\right|, \sigma^{2}$, and $\sigma_{f}^{2}$. The branch metrics (18) are valid in the case of any linear modulation and any (Raylegh, Rice, or AWGN) channel. However, they may be significantly simplified in the following special cases.

\section{A. Equal Energy Signals}

We now concentrate on the special case of equal energy signals, such as PSK modulations in the absence of intersymbol interference. Eliminating from (15) the terms which do not depend on the hypothetical code vector $\tilde{\boldsymbol{c}}$, because the code sym-

$$
\begin{aligned}
\Lambda_{K}(\tilde{\boldsymbol{c}}) & \simeq-\sigma^{2} \ln \left(|\tilde{\boldsymbol{c}}|^{2}+\frac{1}{\gamma}\right)+\frac{1}{2} \frac{\left|\tilde{\boldsymbol{c}}^{H} \boldsymbol{x}\right|^{2}-\frac{\left|\eta_{f}\right|^{2}|\tilde{c}|^{2}}{\gamma}+2 \frac{\left|\eta_{f}\right|\left|\tilde{\boldsymbol{c}}^{H} \boldsymbol{x}\right|}{\gamma}}{\frac{1}{\gamma}+|\tilde{\boldsymbol{c}}|^{2}} \\
& =-\sigma^{2} \ln \left(\sum_{k=0}^{K-1}\left|\tilde{c}_{k}\right|^{2}+\frac{1}{\gamma}\right)+\frac{1}{2} \frac{\left|\sum_{k=0}^{K-1} x_{k} \tilde{c}_{k}^{*}\right|^{2}-\frac{\left|\eta_{f}\right|^{2}}{\gamma} \sum_{k=0}^{K-1}\left|\tilde{c}_{k}\right|^{2}+2 \frac{\left|\eta_{f}\right|}{\gamma}\left|\sum_{k=0}^{K-1} x_{k} \tilde{c}_{k}^{*}\right|}{\frac{1}{\gamma}+\sum_{k=0}^{K-1}\left|\tilde{c}_{k}\right|^{2}}
\end{aligned}
$$

$$
\begin{aligned}
\Delta_{n}(\tilde{\boldsymbol{c}}) & \triangleq \Lambda_{n+1}(\tilde{\boldsymbol{c}})-\Lambda_{n}(\tilde{\boldsymbol{c}}) \\
& =-\sigma^{2} \ln \left(\frac{\sum_{k=0}^{n}\left|\tilde{c}_{k}\right|^{2}+\frac{1}{\gamma}}{\sum_{k=0}^{n-1}\left|\tilde{c}_{k}\right|^{2}+\frac{1}{\gamma}}\right)+\frac{1}{2} \frac{\left|\sum_{k=0}^{n} x_{k} \tilde{c}_{k}^{*}\right|^{2}-\frac{\left|\eta_{f}\right|^{2}}{\gamma} \sum_{k=0}^{n}\left|\tilde{c}_{k}\right|^{2}+2 \frac{\left|\eta_{f}\right|}{\gamma}\left|\sum_{k=0}^{n} x_{k} \tilde{c}_{k}^{*}\right|}{\frac{1}{\gamma}+\sum_{k=0}^{n}\left|\tilde{c}_{k}\right|^{2}} \\
& -\frac{1}{2} \frac{\left|\sum_{k=0}^{n-1} x_{k} \tilde{c}_{k}^{*}\right|^{2}-\frac{\left|\eta_{f}\right|^{2}}{\gamma} \sum_{k=0}^{n-1}\left|\tilde{c}_{k}\right|^{2}+2 \frac{\left|\eta_{f}\right|}{\gamma}\left|\sum_{k=0}^{n-1} x_{k} \tilde{c}_{k}^{*}\right|}{\frac{1}{\gamma}+\sum_{k=0}^{n-1}\left|\tilde{c}_{k}\right|^{2}}
\end{aligned}
$$


bols have now constant modulus, we have the following expression of the sequence metric

$$
\Lambda_{K}(\tilde{\boldsymbol{c}})=\left|\sum_{k=0}^{K-1} x_{k} \tilde{c}_{k}^{*}\right|^{2}+2 \frac{\left|\eta_{f}\right|}{\gamma}\left|\sum_{k=0}^{K-1} x_{k} \tilde{c}_{k}^{*}\right| .
$$

Adding the term $\left|\eta_{f}\right|^{2} / \gamma^{2}$, independent of $\tilde{\boldsymbol{c}}$, we have the following equivalent sequence metric:

$$
\begin{aligned}
\Lambda_{K}(\tilde{\boldsymbol{c}}) & \sim\left|\sum_{k=0}^{K-1} x_{k} \widetilde{c}_{k}^{*}\right|^{2}+2 \frac{\left|\eta_{f}\right|}{\gamma}\left|\sum_{k=0}^{K-1} x_{k} \tilde{c}_{k}^{*}\right|+\frac{\left|\eta_{f}\right|^{2}}{\gamma^{2}} \\
& =\left(\left|\sum_{k=0}^{K-1} x_{k} \tilde{c}_{k}^{*}\right|+\frac{\left|\eta_{f}\right|}{\gamma}\right)^{2} \sim\left|\sum_{k=0}^{K-1} x_{k} \widetilde{c}_{k}^{*}\right| .
\end{aligned}
$$

In the last step, the fact that the square of a positive quantity is monotonic has been used.

Introducing partial sequence metric, incremental, and truncated-memory branch metrics, we finally obtain

$$
\lambda_{n}(\tilde{\boldsymbol{c}})=\left|\sum_{i=0}^{N-1} x_{n-i} \tilde{c}_{n-i}^{*}\right|-\left|\sum_{i=1}^{N-1} x_{n-i} \tilde{c}_{n-i}^{*}\right|
$$

which evidently does not require any statistical information about the channel, because it does not depend on $\left|\eta_{f}\right|, \sigma_{f}^{2}$, and $\sigma^{2}$. This result coincides with that found in [1] for an AWGN channel, in agreement with the intuition that the channel gain is irrelevant in the detection of equal energy signals.

\section{B. Rayleigh Fading Channels}

The branch metrics in the case of a Rayleigh fading channel may be obtained letting $\left|\eta_{f}\right|=0$ in (18). Accordingly, we have

$$
\begin{aligned}
\lambda_{n}(\tilde{\boldsymbol{c}})= & -\sigma^{2} \ln \left(\frac{\sum_{i=0}^{N-1}\left|\tilde{c}_{n-i}\right|^{2}+\frac{1}{\gamma}}{\sum_{i=1}^{N-1}\left|\tilde{c}_{n-i}\right|^{2}+\frac{1}{\gamma}}\right)+\frac{1}{2} \frac{\left|\sum_{i=0}^{N-1} x_{n-i} \tilde{c}_{n-i}^{*}\right|^{2}}{\frac{1}{\gamma}+\sum_{i=0}^{N-1}\left|\tilde{c}_{n-i}\right|^{2}} \\
& -\frac{1}{2} \frac{\left|\sum_{i=1}^{N-1} x_{n-i} \tilde{c}_{n-i}^{*}\right|^{2}}{\frac{1}{\gamma}+\sum_{i=1}^{N-1}\left|\tilde{c}_{n-i}\right|^{2}} .
\end{aligned}
$$

The evaluation of these branch metrics obviously requires knowledge of $\sigma^{2}$ and $\gamma$ (or, equivalently, $\sigma^{2}$ and $\sigma_{f}^{2}$ ). Since high values of signal-to-noise ratio are typical of Rayleigh fading channels, we may use the approximations $\sigma^{2} \simeq 0$ and $1 / \gamma \simeq 0$, obtaining

$$
\lambda_{n}(\tilde{\boldsymbol{c}})=\frac{\left|\sum_{i=0}^{N-1} x_{n-i} \tilde{c}_{n-i}^{*}\right|^{2}}{\sum_{i=0}^{N-1}\left|\tilde{c}_{n-i}\right|^{2}}-\frac{\left|\sum_{i=1}^{N-1} x_{n-i} \tilde{c}_{n-i}^{*}\right|^{2}}{\sum_{i=1}^{N-1}\left|\tilde{c}_{n-i}\right|^{2}}
$$

which are independent of the channel parameters. Simulation results show that the performance of a receiver based on (23) is equivalent to that of the receiver based on (22), in which $\sigma^{2}$ and $\sigma_{f}^{2}$ are assumed perfectly known. Therefore, the approximation used in the derivation of (23) may be considered valid.

An interesting result we obtained by means of simulation is that, in the case of a Rice fading channel, a receiver based on branch metrics (23) is practically equivalent, in terms of performance, to a receiver based on branch metrics (18) in which $\left|\eta_{f}\right|$, $\sigma_{f}^{2}$ and $\sigma^{2}$ are assumed perfectly known. We could not interpret this result in terms of suitable approximations of (18) for a general Rice fading channel. However, we note that a receiver based on branch metrics (23) would deliver reliable decisions independently of the actual value of the fading gain, provided this gain is slowly varying. This provides some intuitive understanding of the fact that this receiver would work independently of the distribution of the fading gain, though we could not have predicted that its performance is good. For these reasons, the branch metrics (23) will be used in the numerical results for nonequal energy signals and both Rice and Rayleigh fading channels.

\section{AWGN Channels}

For an AWGN channel, the results in [1] may be obtained letting $\sigma_{f}^{2}=0, \gamma=0$ and $\left|\eta_{f}\right|=1$. Multiplying numerator and denominator of each fraction in (18) by $\gamma$, it is immediate to obtain

$$
\lambda_{n}(\tilde{\boldsymbol{c}})=\left|\sum_{i=0}^{N-1} x_{n-i} \tilde{c}_{n-i}^{*}\right|-\left|\sum_{i=1}^{N-1} x_{n-i} \widetilde{c}_{n-i}^{*}\right|-\frac{1}{2}\left|\tilde{c}_{n}\right|^{2}
$$

which coincides with the result in [1].

$$
\begin{aligned}
\lambda_{n}(\tilde{\boldsymbol{c}}) \triangleq & -\sigma^{2} \ln \left(\frac{\sum_{i=0}^{N-1}\left|\tilde{c}_{n-i}\right|^{2}+\frac{1}{\gamma}}{\sum_{i=1}^{N-1}\left|\tilde{c}_{n-i}\right|^{2}+\frac{1}{\gamma}}\right)+\frac{1}{2} \frac{\left|\sum_{i=0}^{N-1} x_{n-i} \tilde{c}_{n-i}^{*}\right|^{2}-\frac{\left|\eta_{f}\right|^{2}}{\gamma} \sum_{i=0}^{N-1}\left|\tilde{c}_{n-i}\right|^{2}+2 \frac{\left|\eta_{f}\right|}{\gamma}\left|\sum_{i=0}^{N-1} x_{n-i} \tilde{c}_{n-i}^{*}\right|}{\frac{1}{\gamma}+\sum_{i=0}^{N-1}\left|\tilde{c}_{n-i}\right|^{2}} \\
& -\frac{1}{2} \frac{\left|\sum_{i=1}^{N-1} x_{n-i} \tilde{c}_{n-i}^{*}\right|^{2}-\frac{\left|\eta_{f}\right|^{2}}{\gamma} \sum_{i=1}^{N-1}\left|\tilde{c}_{n-i}\right|^{2}+2 \frac{\left|\eta_{f}\right|}{\gamma}\left|\sum_{i=1}^{N-1} x_{n-i} \tilde{c}_{n-i}^{*}\right|}{\frac{1}{\gamma}+\sum_{i=1}^{N-1}\left|\tilde{c}_{n-i}\right|^{2}}
\end{aligned}
$$


TABLE I

Summary of THE DeRIVEd BRanch Metrics IN the CASE OF ABSENCE OF CSI

\begin{tabular}{|c|c|c|}
\hline Rayleigh channels & \multirow{3}{*}{ eq. (21) } & \multirow[t]{2}{*}{ eq. (23) } \\
\hline Rice channels & & \\
\hline AWGN channels & & eq. $(24)$ or $(23)$ \\
\hline & Equal energy signals & Non-equal energy signals \\
\hline
\end{tabular}

Interestingly, simulation results show that branch metrics (23) may also be used in this case with a minor energy loss, with respect to the metrics (24), of a fraction of decibels only. This fact confirms the correctness of the interpretation given at the end of the previous section.

\section{Summary of NSD Without CSI}

In Table I, the expression of the proposed branch metrics in each considered case is summarized. In all cases, a knowledge of channel statistics is not required.

\section{NUMERICAL RESULTS}

The performance of the proposed detection algorithms is assessed by means of computer simulations in terms of bit error rate (BER) versus $E_{b} / N_{0}, E_{b}$ being the average received signal energy per information bit.

As in [1] and [2], the state-complexity of the proposed detection schemes may be limited by reduced-state sequence detection RSSD [21], [22]. This technique allows us to choose independently the two parameters: phase memory $N$ and number of states $S$ of the Viterbi algorithm. Hence, the number of states may be limited without excessively reducing the value of $N$. In order to compute the branch metrics (6), (21), (23), or (24) in a reduced trellis, the necessary symbols not included or not completely specified in the state definition are found in the survivor history according to per-survivor processing (PSP) [23]. Receivers with $S=1$ and $S=4$ are considered. We note that, in the limiting case of $S=1$, the trellis diagram degenerates and symbol-by-symbol detection with decision feedback is performed.

The transmitter and receiver filters are assumed to have equal square-root raised-cosine frequency response with rolloff 0.5 . Each burst is composed of a preamble and an information field of 100 symbols. CSI-based receivers use a preamble of 10 symbols. These known symbols are used for estimating the fading gain $|f|$ using a simple method based on the sample mean. A more refined estimation may be conceived but, in the case of a slow fading channel, the used method gives a receiver performance equal to that achievable with a perfect knowledge of $|f|$. For the receivers without CSI, a preamble of $N-1$ symbols is used. These symbols are necessary to initialize state and branch metrics of the Viterbi algorithm. In both cases of presence or absence of CSI, a postamble of 1 symbol for the receivers with $S=4$ is present in order to correctly terminate the Viterbi algorithm.

In the case of 16-QAM, quadrant differential encoding, as described in [1], is used. The performance of the proposed receivers with $S=4$ and $N=3$ for Rice fading with $K_{R}=10$

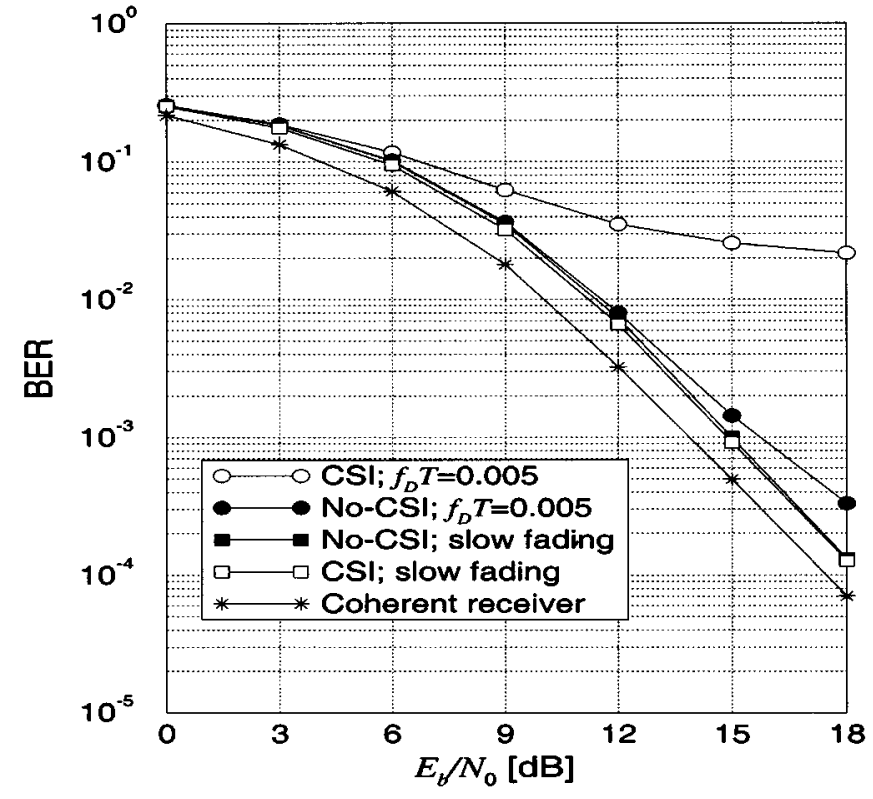

Fig. 2. BER of the proposed receivers with and without CSI, $S=4$ and $N=$ 3 , for differentially encoded 16-QAM and Rice fading with $K_{R}=10 \mathrm{~dB}$. The performance of an ideal coherent receiver is also shown for comparison.

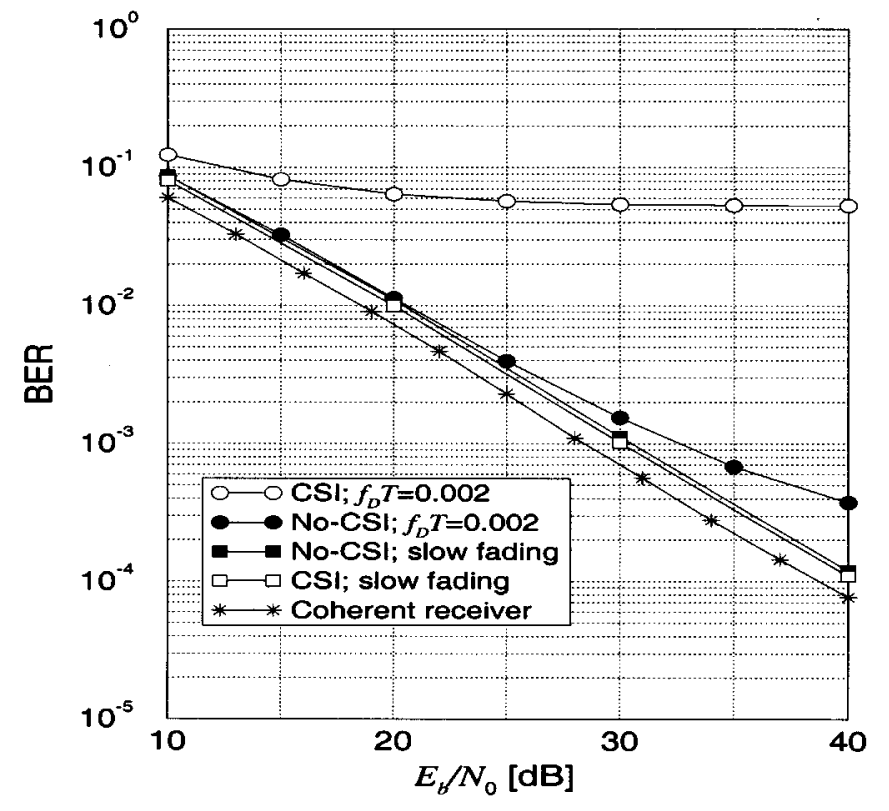

Fig. 3. BER of the proposed receivers with and without CSI, $S=4$ and $N=$ 3 , for differentially encoded 16-QAM and Rayleigh fading. The performance of an ideal coherent receiver is also shown for comparison.

$\mathrm{dB}$ and Rayleigh fading is shown in Figs. 2 and 3, respectively. The performance of an ideal coherent receiver, i.e., a receiver which perfectly knows the fading coefficient $f$ and the channel phase $\theta$, is also shown for comparison. It may be observed that the proposed receivers exhibit a moderate performance loss with respect to the ideal coherent detector. Furthermore, the receivers with and without CSI are practically equivalent.

The robustness of these receivers in the case of time-varying fading channels is also investigated. The fading autocorrelation function is assumed equal to $J_{0}\left(2 \pi f_{D} \tau\right)$, where $J_{0}(\cdot)$ is the Bessel function of zeroth order, according to an isotropic scattering model [24]. The fading speed depends on the normalized 


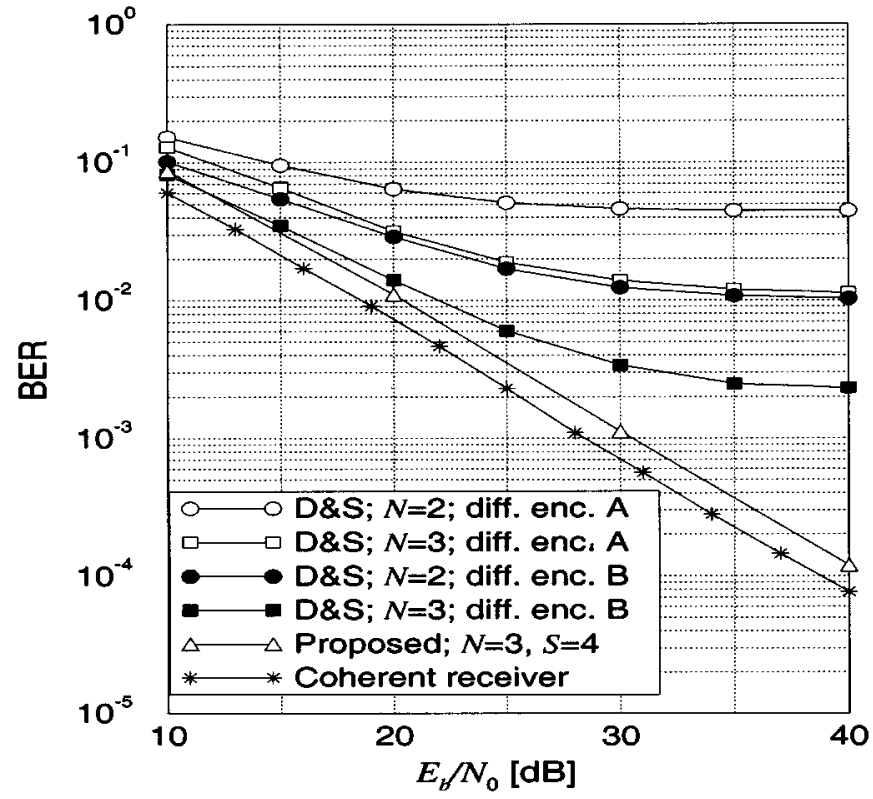

Fig. 4. BER of the proposed receiver without CSI, $S=4$ and $N=3$, for differentially encoded 16-QAM and slow Rayleigh fading, and comparison to that of the receivers in [14] (D\&S).

Doppler band $f_{D} T$, which is set to the values 0.005 and 0.002 for Rice and Rayleigh fading, respectively. From Figs. 2 and 3, we may conclude that, in the presence of a time-varying fading, the receivers which operate without CSI are clearly more robust than those with CSI, whose performance rapidly degrades. We have verified that this behavior is not due to an insufficient quality of estimation of the fading amplitude by providing an idealized receiver with perfect initial CSI. The high error floor exhibited by the CSI-based receiver is mainly due to the significant time variation of the fading amplitude within the data burst, and due to the fact that the considered receivers do not employ a tracking subsystem in order to adaptively estimate the channel gain value.

In Fig. 4, the performance of the proposed receiver without CSI, $S=4$ and $N=3$ is compared to that of the receivers proposed in [14] by Divsalar and Simon (D\&S) in the case of differentially encoded 16-QAM and a Rayleigh fading channel. The proposed receiver performs better and does not exhibit an error floor which, on the contrary, characterizes the performance of the receivers in [14], even in the case of a slow fading channel and for both the quadrant differential encoding rule here considered (case A) and the differential encoding rule considered in [14] (case B). The existence of this error floor is theoretically proved in the Appendix. Note that the differential encoding rule, adopted in [14], modifies an original $M$-point square QAM constellation into a nonsquare one characterized by a larger number of points.

Finally, the performance under dynamic channel conditions has been investigated. Two types of time-varying phase models are considered. The first one is the well-known stochastic model of phase noise. Accordingly, the phase $\theta$ of the received signal is modeled as a time-continuous Wiener process with incremental variance over a symbol interval equal to $\sigma_{\Delta}^{2}$. The second one is a deterministic model of a frequency offset $\nu$ which, as an

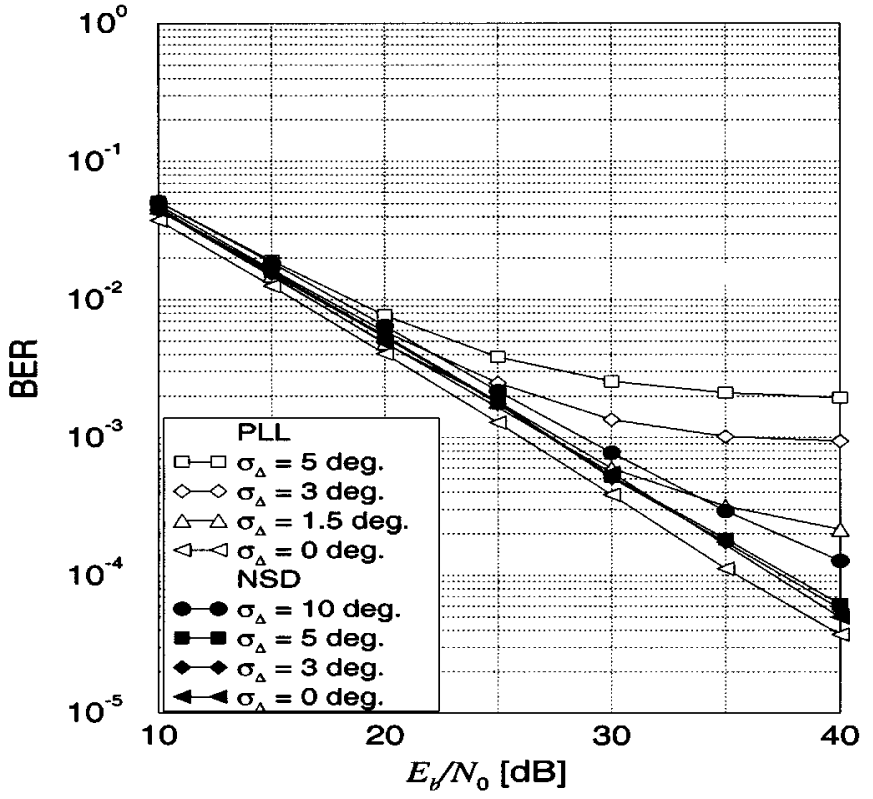

Fig. 5. BER of the proposed receiver without CSI, $S=1$ and $N=4$, for differentially encoded QPSK, slow Rayleigh fading, various values of phase noise standard deviation (black marks) and comparison with a coherent receiver based on a decision-directed PLL (white marks).

example, may be due to a Doppler shift in a mobile to satellite system with a low earth orbit (LEO) satellite.

Fig. 5 compares the performance of the proposed noncoherent receiver with $N=4$ and $S=1$, for differentially encoded QPSK and a slow Rayleigh fading channel, to that of a coherent receiver based on a second-order maximum-likelihood decision-directed PLL. In this case, the coherent receiver does not need any knowledge about the fading coefficient, and is based on a symbol-by-symbol receiver followed by a differential decoder. For the considered burst-mode transmission scheme, the PLL is ideally reinitialized at the beginning of each data burst with the correct phase value; hence, it starts in the lock-in condition. In the figure, the value of the PLL noise equivalent bandwidth has been optimized by simulation for each value of $E_{b} / N_{0}$. As we may note, a phase noise standard deviation up to $5^{\circ}$ does not significantly degrade the performance of the proposed noncoherent receiver. This may be also noted in Fig. 6 which shows, for both receivers, the signal-to-noise ratio for a BER of $10^{-3}$, as a function of the phase noise standard deviation. Unlike the proposed receivers, the PLL-based ones are affected by unavoidable losses of the lock-in condition within the data burst for high phase noise.

The proposed noncoherent receivers are also robust to frequency offsets, as may be observed in Fig. 7 for differentially encoded QPSK and a slow Rayleigh fading channel (receiver with $N=4$, and $S=1$ ). Frequency offsets $\nu$, normalized to the symbol frequency $1 / T$, up to $\nu T=10^{-2}$ do not entail appreciable degradation.

\section{CONCLUSION}

Noncoherent sequence detection is establishing itself as an attractive alternative to PLL-based coherent detection in applications where phase or frequency instabilities are significant and 


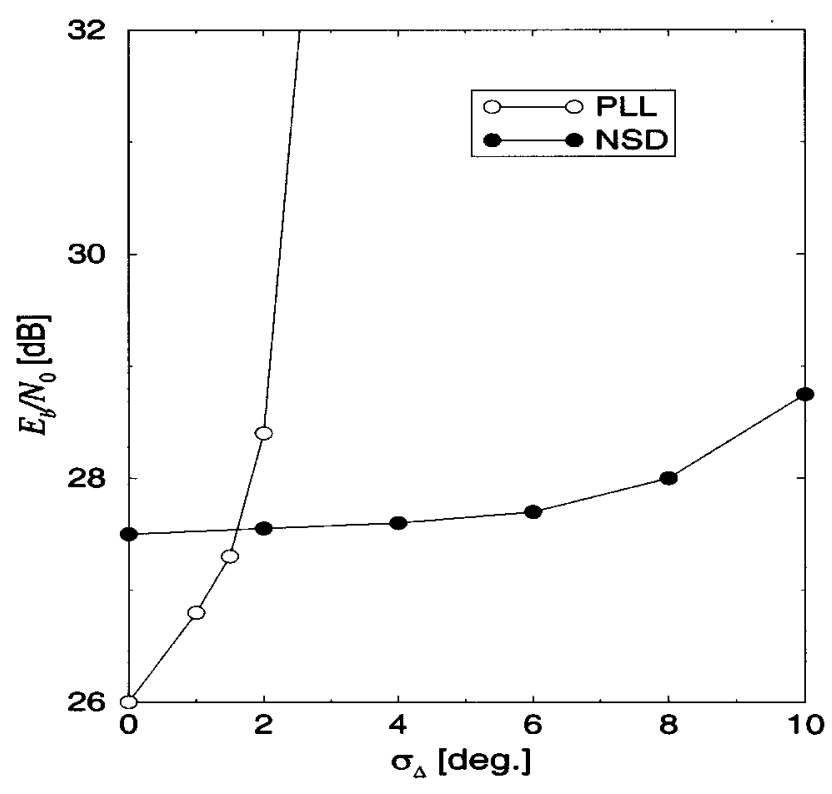

Fig. 6. $E_{b} / N_{0}$ as a function of the phase noise standard deviation for BER equal to $10^{-3}$ of the proposed receiver without CSI, $S=1$ and $N=4$, for differentially encoded QPSK, slow Rayleigh fading (black marks), and comparison to a coherent receiver based on a decision-directed PLL (white marks).

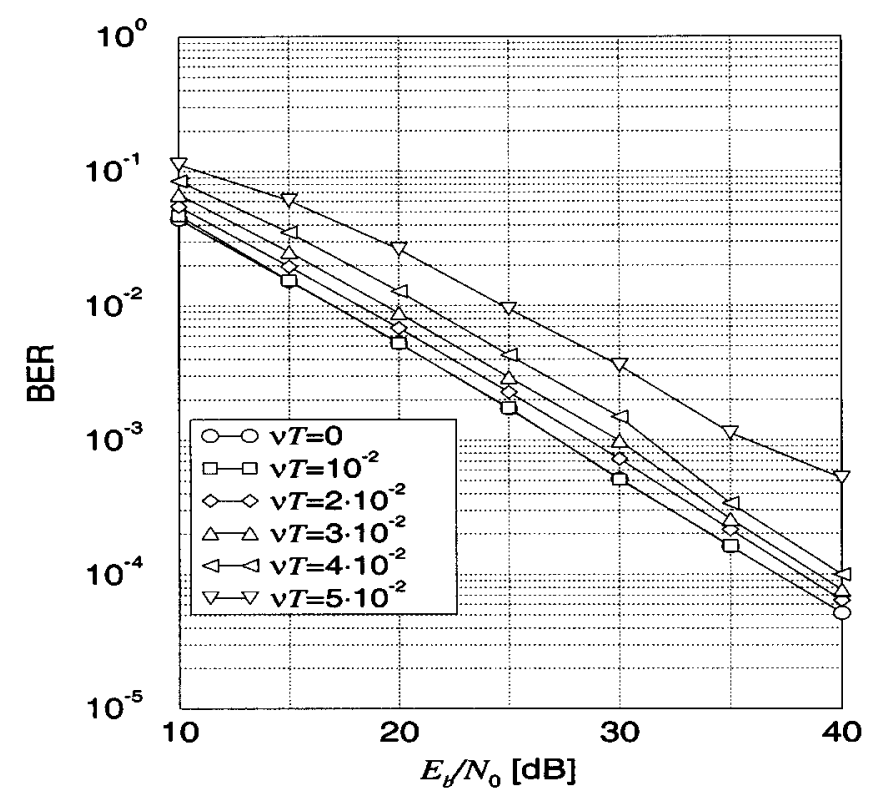

Fig. 7. BER of the proposed receiver without CSI, $S=1$ and $N=4$, for differentially encoded QPSK, slow Rayleigh fading, and various values of the normalized frequency offset.

burst-mode transmissions. This paper extends noncoherent sequence detection to the case of slowly fading channels. Very effective detection strategies have been presented which do not require any knowledge of channel statistics and achieve a performance close to that of ideal coherent detection for Rice fading with any factor (from AWGN to Rayleigh fading). Multiamplitude modulation formats, such as 16-QAM, have been explicitly considered. The proposed NSD schemes derived in the absence of CSI are very robust to time-varying fading as well as phase or frequency instabilities, and are clearly more robust than schemes derived under the assumption of perfect CSI.

\section{APPENDIX}

In [14], MSDD schemes for slow Rayleigh fading and multiamplitude modulation formats have been proposed. The receiver operates an exhaustive search over a block of $N$ symbols, based on the metric

$$
\begin{aligned}
\eta(\tilde{\boldsymbol{c}}) \triangleq & -\sigma^{2} \ln \left(\sigma_{f}^{2} \sum_{i=0}^{N-1}\left|\tilde{c}_{k-i}\right|^{2}+\sigma^{2}\right) \\
+ & \frac{\sigma_{f}^{2}\left|\sum_{i=0}^{N-1} x_{k-i} \tilde{c}_{k-i}^{*}\right|^{2}}{\sigma^{2}+\sigma_{f}^{2} \sum_{i=0}^{N-1}\left|\tilde{c}_{k-i}\right|^{2}}
\end{aligned}
$$

where $\tilde{\boldsymbol{c}}=\left(\tilde{c}_{k-N+1}, \cdots, \tilde{c}_{k-1}, \tilde{c}_{k}\right)$ is the hypothetical block of $N$ code symbols. In this appendix, we prove the existence of an irreducible error probability, or error floor, for this receiver.

The performance of a receiver exhibits an error floor if, even for $E_{b} / N_{0} \rightarrow \infty$, i.e., in the absence of noise, receiver decisions may be affected by some errors. In the absence of noise, we have $\sigma^{2}=0$ and $x_{k}=c_{k} f e^{j \theta}$. Substituting in (A1), we obtain

$$
\eta(\tilde{\boldsymbol{c}})=|f|^{2} \frac{\left|\sum_{i=0}^{N-1} \tilde{c}_{k-i}^{*} c_{k-i}\right|^{2}}{\sum_{i=0}^{N-1}\left|\tilde{c}_{k-i}\right|^{2}} .
$$

A receiver exhibits an error floor if it is possible to find a transmitted block $\boldsymbol{c}$ and an admissible competitor block $\tilde{\boldsymbol{c}}$, such that, in the absence of noise,

$$
\eta(\tilde{c}) \geq \eta(\boldsymbol{c})
$$

If $\eta(\tilde{c})>\eta(\boldsymbol{c})$, the receiver erroneously selects the sequence $\tilde{\boldsymbol{c}}$ If $\eta(\tilde{\boldsymbol{c}})=\eta(\boldsymbol{c})$, the receiver chooses randomly between the two sequences. As a consequence, an error is made with probability $1 / 2$.

In this paper, in the case of multiamplitude modulations, such as $M$-QAM, a quadrant differential encoding is considered [1]. A different differential encoding rule is adopted in [14] which, however, modifies an original square QAM constellation into a nonsquare one characterized by a larger number of points. Following this differential encoding rule, the code symbol $c_{k}$ is related to the information symbol $a_{k}$ by [14]

$$
c_{k}=a_{k} e^{j \arg \left\{c_{k-1}\right\}} .
$$

We now show the existence of an error floor for the metric (A1) and both differential encoding rules.

We consider the case of a square QAM constellation and, in general, constellations which include the points $a_{k}=1+j$ and $a_{k}=3+3 j$. In the case of quadrant differential encoding [1], if

$$
\begin{aligned}
& a_{k}=a_{k-1}=\cdots=a_{k-N+1}=1+j=\sqrt{2} e^{j(\pi / 4)} \\
& \tilde{a}_{k}=\tilde{a}_{k-1}=\cdots=\tilde{a}_{k-N+1}=3+3 j=3 \sqrt{2} e^{j(\pi / 4)}
\end{aligned}
$$

and code symbols $c_{k-N}$ and $\tilde{c}_{k-N}$ belong to the first quadrant, we have

$$
\begin{aligned}
& c_{k}=c_{k-1}=\cdots=c_{k-N+1}=1+j=\sqrt{2} e^{j(\pi / 4)} \\
& \tilde{c}_{k}=\tilde{c}_{k-1}=\cdots=\tilde{c}_{k-N+1}=3+3 j=3 \sqrt{2} e^{j(\pi / 4)}
\end{aligned}
$$


and

$$
\begin{aligned}
\eta(\boldsymbol{c}) & =|f|^{2} \frac{\left|\sum_{i=0}^{N-1} c_{k-i} c_{k-i}^{*}\right|^{2}}{\sum_{i=0}^{N-1}\left|c_{k-i}\right|^{2}} \\
& =|f|^{2} \sum_{i=0}^{N-1}\left|c_{k-i}\right|^{2}=2 N|f|^{2} \\
\eta(\tilde{\boldsymbol{c}}) & =|f|^{2} \frac{\left|\sum_{i=0}^{N-1} c_{k-i} \tilde{c}_{k-i}^{*}\right|^{2}}{\sum_{i=0}^{N-1}\left|\tilde{c}_{k-i}\right|^{2}} \\
& =|f|^{2} \frac{(6 N)^{2}}{18 N}=2 N|f|^{2} .
\end{aligned}
$$

Therefore, $\eta(\tilde{\boldsymbol{c}})=\eta(\boldsymbol{c})$, and the receiver chooses randomly between the two sequences.

An identical conclusion may be reached adopting the encoding rule (A4). In fact, considering symbols $a_{k-i}$ and $\tilde{a}_{k-i}$, $i=0,1, \cdots, N-1$, given in (A5) and (A6) and assuming

$$
\arg \left\{c_{k-N}\right\}=\arg \left\{\tilde{c}_{k-N}\right\}
$$

we have

$$
\arg \left\{c_{k-i}\right\}=\arg \left\{\tilde{c}_{k-i}\right\}, \quad i=0,1, \cdots, N-1
$$

and, therefore,

$$
c_{k-i} \tilde{c}_{k-i}^{*}=\left|c_{k-i}\right|\left|\tilde{c}_{k-i}\right|, \quad i=0,1, \cdots, N-1 .
$$

As a consequence, noting that $\left|c_{k-i}\right|=\left|a_{k-i}\right|$ and $\left|\tilde{c}_{k-i}\right|=$ $\left|\tilde{a}_{k-i}\right|$, we again have

$$
\eta(\boldsymbol{c})=\eta(\tilde{\boldsymbol{c}})=2 N|f|^{2}
$$

Due to these undistinguishable sequences, the performance of the receiver in [14] is characterized by an error floor which is not caused by the presence of fading. In fact, being the fading constant over the transmitted data burst, it does not influence the receiver performance in the absence of noise. For increasing values of $N$, the ratio between the undistinguishable sequences and the number of possible sequences decreases, thus decreasing the value of the error floor.

\section{REFERENCES}

[1] G. Colavolpe and R. Raheli, "Noncoherent sequence detection," IEEE Trans. Commun., vol. 47, pp. 1376-1385, Sept. 1999.

[2] — , "Noncoherent sequence detection of continuous phase modulations," IEEE Trans. Commun., vol. 47, pp. 1303-1307, Sept. 1999.

[3] P. Y. Kam, "Maximum-likelihood carrier phase recovery for linear suppressed-carrier digital data modulations," IEEE Trans. Commun., vol. COM-34, pp. 522-527, June 1986.
[4] H. Leib and S. Pasupathy, "The phase of a vector perturbed by Gaussian noise and differentially coherent receivers," IEEE Inform. Theory, vol. 34, pp. 1491-1501, Nov. 1988.

[5] F. Edbauer, "Bit error rate of binary and quaternary DPSK signals with multiple differential feedback detection," IEEE Trans. Commun., vol. 40, pp. 457-460, Mar. 1992

[6] P. Y. Kam, S. S. Ng, and T. S. Ng, "Optimum symbol-by symbol detection of uncoded digital data over the Gaussian channel with unknown carrier phase," IEEE Trans. Commun., vol. 42, pp. 2543-2551, Aug. 1994.

[7] S. G. Wilson, J. Freebersyser, and C. Marshall, "Multi-symbol detection of $M$-DPSK," in Proc. Global Commun. Conf. (GLOBECOM'89), Nov. 1989, pp. 1692-1697.

[8] D. Divsalar and M. K. Simon, "Multiple-symbol differential detection of MPSK," IEEE Trans. Commun., vol. 38, pp. 300-308, Mar. 1990.

[9] H. Leib and S. Pasupathy, "Noncoherent block demodulation of PSK," in Proc. Veh. Technol. Conf. (VTC'90), May 1990, pp. 407-411.

[10] M. K. Simon, S. M. Hinedi, and W. C. Lindsey, Digital Communication Techniques. Englewood Cliffs, NJ: Prentice-Hall, 1995.

[11] D. Divsalar, M. K. Simon, and M. Shahshahani, "The performance of trellis-coded MDPSK with multiple symbol detection," IEEE Trans. Commun., vol. 38, pp. 1391-1403, Sept. 1990.

[12] Y. Kofman, E. Zehavi, and S. Shamai, "nd-convolutional codes-Part I: Performance analysis," IEEE Trans. Inform. Theory, vol. 43, pp. 558-575, Mar. 1997.

[13] — , "nd-convolutional codes-Part II: Structural analysis," IEEE Trans. Inform. Theory, vol. 43, pp. 576-589, Mar. 1997.

[14] D. Divsalar and M. K. Simon, "Maximum-likelihood differential detection of uncoded and trellis coded amplitude phase modulation over AWGN and fading channels-Metrics and performance," IEEE Trans. Commun., vol. 42, pp. 76-89, Jan. 1994.

[15] M. K. Simon and D. Divsalar, "Multiple symbol partially coherent detection of MPSK," IEEE Trans. Commun., vol. 42, pp. 430-439, Feb./Mar./Apr. 1994.

[16] D. Raphaeli, "Noncoherent coded modulation," IEEE Trans. Commun., vol. 44, pp. 172-183, Feb. 1996.

[17] J. Proakis, Digital Communications, 2nd ed. New York: McGraw-Hill, 1989.

[18] G. Colavolpe and R. Raheli, "Theoretical analysis and performance limits of noncoherent sequence detection of coded PSK," IEEE Trans. Inform. Theory, vol. 46, pp. 1483-1494, July 2000.

[19] P. Castoldi and R. Raheli, "On recursive optimal detection of linear modulations in the presence of random fading," European Trans. Telecommun., vol. 9, no. 2, pp. 209-220, Mar.-Apr. 1998.

[20] S. L. Marple, Digital Spectral Analysis with Applications. Englewood Cliffs, NJ: Prentice-Hall, 1987.

[21] M. V. Eyuboğlu and S. U. H. Qureshi, "Reduced-state sequence estimation with set partitioning and decision feedback," IEEE Trans. Commun., vol. 36, pp. 13-20, Jan. 1988.

[22] P. R. Chevillat and E. Eleftheriou, "Decoding of trellis-encoded signals in the presence of intersymbol interference and noise," IEEE Trans. Commun., vol. 37, pp. 669-676, July 1989.

[23] R. Raheli, A. Polydoros, and C. K. Tzou, "Per-survivor processing: A general approach to MLSE in uncertain environments," IEEE Trans. Commun., vol. 43, pp. 354-364, Feb.-Apr. 1995.

[24] R. Clarke, "A statistical theory of mobile radio reception," Bell Syst. Tech. J., vol. 47, pp. 957-1000, 1968.

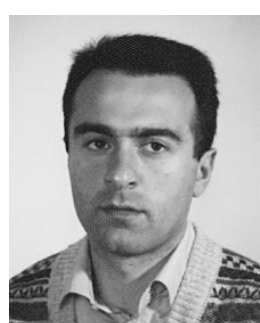

Giulio Colavolpe (S'97) was born in Cosenza, Italy, in 1969. He received the Dr.Ing. degree in telecommunications engineering (cum laude) from the University of Pisa, Italy, in 1994 and Doctoral degree in information technology from the University of Parma, Italy, in 1998. From December 1997 to October 1999, he was a Research Associate at the University of Parma. Since November 1999, he has been a Research Professor at the University of Parma. His main research interests include digital transmission theory, channel coding, and signal processing. 


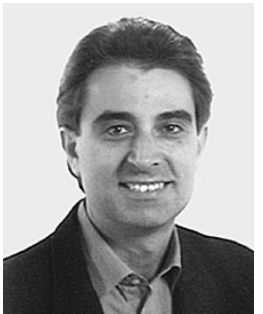

Riccardo Raheli (M'87) was born in Alezio (Lecce), Italy, in 1957. He received the Dr.Ing. degree (Laurea) from the University of Pisa, Italy, in 1983, the Master of Science degree from the University of Massachusetts, Amherst, USA, in 1986, and the Doctoral degree (Perfezionamento) from the Scuola Superiore di Studi Universitari e di Perfezionamento, Pisa, Italy, in 1987, all in electrical engineering.

From 1986 to 1988, he was a Project Engineer with

Siemens Telecomunicazioni, Milan, Italy. From 1988 to 1991, he was a Research Professor at the Scuola Superiore di Studi Universitari e di Perfezionamento S. Anna, Pisa, Italy. In 1990, he was a Visiting Assistant Professor at the University of Southern California, Los Angeles, USA. Since 1991, he has been a Faculty Member at the University of Parma, Italy, where he is currently an Associate Professor of Telecommunications. Since 1999, he has also served on the Editorial Board of the IEEE TRANSACTIONS ON COMMUNICATIONS as an Editor for Detection, Equalization and Coding. His main research interests include digital transmission theory, sequence detection techniques, signal processing and adaptive algorithms for communications. His research activity has led to over fifty scientific publications in international journals and conference proceedings and a few industrial patents. 